\title{
Integration of Steerable Smart Antennas to IETF 6TiSCH Protocol for High Reliability Wireless loT Networks
}

This paper was downloaded from TechRxiv (https://www.techrxiv.org).

\section{LICENSE}

CC BY 4.0

SUBMISSION DATE / POSTED DATE

$30-01-2021 / 26-07-2021$

\section{CITATION}

Kulcu, Sercan; Gormus, Sedat; Jin, Yichao (2021): Integration of Steerable Smart Antennas to IETF 6TiSCH Protocol for High Reliability Wireless loT Networks. TechRxiv. Preprint.

https://doi.org/10.36227/techrxiv.13668992.v2

$\mathrm{DOI}$

10.36227/techrxiv.13668992.v2 


\title{
Integration of Steerable Smart Antennas to IETF 6TiSCH Protocol for High Reliability Wireless IoT Networks
}

\author{
Sercan Kulcu, Sedat Gormus, Yichao Jin
}

\begin{abstract}
Steerable directional antennas are increasingly utilised to improve the overall performance of the traditional wireless sensor networks. Steerable directional antenna based networking solutions increase the network capacity by providing a longer range of transmission and reduced interference as compared to networking solutions with omni-directional antennas. However, the use of smart antennas requires complex algorithms and such algorithms may not be easily leveraged in low power Internet of Things (IoT) networks. This study presents mechanisms for integrating low complexity smart antenna solutions into IETF 6TiSCH protocol with the aim of creating scalable and reliable industrial IoT networks. The solution defines extensions to MAC layer and scheduling mechanisms of IETF 6TiSCH protocol to enable its seamless integration with low complexity steerable smart antennas. The results of this study show that smart antenna enabled 6TiSCH protocol stack outperforms the legacy 6TiSCH stack in terms of data delivery performance especially in high density scenarios.
\end{abstract}

Index Terms-6TiSCH, Network Formation, Smart Antennas, Wireless Sensor Networks.

\section{INTRODUCTION}

$\mathbf{I}$ OT networks are envisaged to be employed in a wide range of applications such as habitat monitoring, disaster relief, smart metering, asset tracking, etc. Such wireless IoT networks are spread over a large area and they are expected to operate for years using limited energy resources. Such networks are generally composed of low power, low cost devices and characterised by intermittent connectivity. Therefore, IoT applications require computationally-light, and energyefficient protocols that can run on such constrained devices [1].

The data generated by the IoT applications are generally delivered to a central node over a multi-hop wireless mesh network for analysis and decision making. This requires the nodes in the network make simultaneous data transfer. However, such data transmissions create in-network interference and lead to packet drops. This is especially true when the network density is high and available bandwidth resources are limited which is the case for most of the low power IoT networks. There are protocols and mechanisms to address in-network interference issues, but such solutions have limited spatial reuse capabilities which may limit the network throughput [2].

Sercan Kulcu and Sedat Gormus are with the Department of Computer Engineering, Karadeniz Technical University, Trabzon, Turkey

e-mail: \{sedatgormus, sercankulcu\}@ ceng.ktu.edu.tr.

Yichao Jin is with Bristol Research and Innovation lab. Toshiba Europe Ltd. Bristol, U.K. e-mail: yichao.jin@toshiba-bril.com
On the other hand, packet drops due to interference can be mitigated by utilising directional antenna systems instead of omni-directional communications [3].

IEEE 802.15.4 standard is the accepted technology for low-power sensor networks [4] which shares the same unlicensed band with other technologies such as WiFi. Therefore, the interference caused by these technologies decrease the performance of IEEE 802.15.4 networks [5]. Electronically steerable parasitic array radiator (ESPAR) antennas may provide a solution for mitigating interference related performance degradation in IEEE 802.15.4 based sensor networks [6]. ESPAR antenna can steer the radiation pattern electronically on the fly towards the desired direction [7]. However, using dynamically steerable directional antennas in 802.15 .4 based WSNs, increases the complexity of the protocol stack design. Furthermore, the wireless channel conditions are time-varying requiring additional adaptive mechanisms to take advantage of directional communication [8].

The IEEE 802.15.4e Time-slotted Channel Hopping (TSCH) MAC layer is adopted for addressing the high reliability requirements of industrial applications. TSCH provides multichannel and synchronized communication to enable high reliability one-hop communication. IETF Working Group 6TiSCH builds a mesh networking solution on top of the TSCH MAC layer to create Industrial IoT networks [9]. 6TiSCH networks build a schedule for the nodes to follow. Nodes decide their activities by following this schedule, that enables them to go into low energy modes during the unscheduled instances [10].

The configurable directional communication opens up a wealth of opportunities. Particularly, it is of interest to integrate such low complexity antenna solutions to Time Synchronized Medium Access Control (MAC) mechanism where the antenna direction can be configured as a channel access parameter. In this work, we introduce Steerable Antenna Agnostic 6TiSCH Solution (SAA6) which integrates a low complexity steerable antenna solution to IETF 6 TiSCH protocol. However, the use of steerable antennas is not limited to 6TiSCH networks. It can be used in other solutions like GALLOP protocol [11] that has very low and deterministic latency, very high reliability and low-overhead signaling mechanism for large networks by providing multi-path communication.

To exploit the benefits of directional antennas, 6TiSCH protocol stack must be redesigned. The redesigned protocol requires the direction information to a destination/source node while transmitting/receiving to improve communication reliability. Voigt et al. showed that the Received Signal Strength 
Indicator (RSSI) can be used to accurately estimate the best transmit/receive direction [12].

The proposal in this paper has the following novel features:

- A novel autonomous steerable antenna aware scheduling mechanism for a faster neighbor discovery.

- RSSI based optimal transmit direction finding algorithm to suppress interference within the network.

- A method to reduce shared cell collisions by utilising directional communication which has a significant impact on 6TiSCH network scalability.

This paper presents the relevant background work in smart antenna systems, and scheduling algorithms in Section II. Section III introduces the developed antenna model. The proposed solution and analysis are presented in Section IV. Performance evaluation and test results are given in Section V. Finally, Section VI outlines the concluding remarks and possible future directions.

\section{RELATED WORK}

\section{A. Smart Antenna Systems}

Smart antennas can be classified as switched beam, and adaptive array antennas. A switched beam antenna has several fixed beam patterns which can behave as an omni-directional antenna to broadcast a frame [13]. On the other hand, adaptive array antennas can steer the beam in any desired direction and cancel interfering signals from undesired directions. But, they can not transmit omni-directionally. Therefore, such antenna solutions require new mechanisms to integrate them into 6TiSCH protocol stack.

ESPAR antenna is one of the most popular and easily integrated structure providing beam steering capability. The ESPAR antenna has a single radiating active element surrounded by a series of passive elements connected to variable reactants. It is possible to change the direction of the main antenna beam by changing the values of the passive elements electronically [6]. ESPAR antennas can be used with WSN nodes as shown in [14]. ESPAR antennas can achieve a $360^{\circ}$ beam scan with different discrete angular steps (30 [15], $45^{\circ}$ [6], 60 ${ }^{\circ}$ [14], [16]). Such antennas have different gains depending on their designs (7.5dBi [16], $8 d B i$ [14], 9dBi [15]).

\section{B. 6TiSCH Protocol}

6TiSCH Working Group (WG) proposes a set of specifications to provide IPv6 compliant networking solution over the TSCH mode [9]. Two steps must be completed for an operational 6TiSCH network: As the first step, a globally synchronized network is built by exchanging Enhanced Beacon (EB) frames. To be able to communicate in a scheduled time slot, nodes must be tightly synchronized. TSCH network coordinator $^{1}$ broadcasts $\mathrm{EB}$ frames to advertise the presence of the network. Nodes follow a scan process to associate to the network. After receiving an EB frame, node learns the minimal schedule and gets synchronized to the network. Following the synchronisation, the nodes in the network are

${ }^{1}$ RPL Root node takes up the network coordinator role in 6 TiSCH networks [17]. configured as a directed tree rooted at the sink by exchanging DODAG Information Object (DIO) frames as defined by the RPL routing protocol [17]. After this step the node has the necessary information to interact with the rest of the network. Here, it is assumed that the join process does not require a subsequent authentication step.

TSCH splits the time into time slots, and multiple time slots form a slotframe which repeats over time. A slotframe is represented as a matrix where each cell being defined by a pair of time slot and channel offset. In a 6TiSCH network each node is responsible for creating a schedule that defines their interaction with the neighboring nodes. During each time slot a node may perform the following actions; transmit, listen, or sleep. All communications are orchestrated by this schedule that contains two types of cells: (1) Shared cell allows a slotted-aloha with contention. For example, Hard cells in 6TiSCH are often shared cell type for signaling purpose. Once a node joins the network, it communicates in shared cell to start a negotiation with its neighbors. (2) Dedicated cell allows interference-free and collision-free communication to provide high reliability. These resources are allocated by a mechanism in a centralized, distributed or autonomous way [18].

In WSNs, nodes are deployed without any prior information about the neighbors and the network topology. Therefore, neighbor discovery process is the first step of self-organization. During this process, each node identifies all of the nodes which it can communicate with directly [19]. To achieve this goal, each node broadcasts periodic neighbor discovery messages. Neighbors should be discovered as quickly as possible to create an accurate picture of the local topology from the nodes point of view.

Neighbor discovery process is trivial when omni-directional antennas are used, since a broadcast transmission can ideally reach all of the neighbors at once. However, use of directional communication makes the neighbor discovery more challenging [19], [20] because of the following reasons: (1) Each node should estimate when and where to point its antenna to discover each potential neighbor, (2) The limited coverage of the directional beam requires the steps to be repeated until the whole azimuth is covered. To be able to discover a neighbor node, the receiving node must be listening at the right time, on the right channel and in the right direction with the transmitter node. This requires additional mechanisms to minimize the synchronization time of the 6TiSCH networks.

\section{6TiSCH Scheduling Mechanisms}

The IEEE 802.15.4e-TSCH standard does not define specific scheduling policies for the transmission of the control frames. The 6TiSCH WG addresses such scheduling challenges by relying on a "minimal profile" standardized in the RFC8180 [21]. This profile defines a common schedule for all the nodes during the network formation. 6TiSCH handles the scheduling functionality via two components: the 6TiSCH Operation Sublayer (6top) Protocol (6P) and the Scheduling Function (SF). 6top Protocol (6P) is a negotiation protocol that allows neighbor nodes to allocate cells between each other [22]. Scheduling Function (SF) is a separate entity and 
supports different cell allocation policies for different network scenarios. Furthermore, 6TiSCH WG standardizes the Minimal Scheduling Function (MSF) for interoperability purposes [9].

Naturally, the scheduling of the control messages plays a key role in the formation phase. The majority of the research efforts focus on the resource allocation in order to guarantee delivery of the messages after the initial formation phase [18], [23]. Most of these solutions assume that all of the nodes are part of the network and have discovered the optimal routes to the sink. The algorithms related to EB scheduling are generally shared cell based solutions and they assume that nodes have only omni-directional antennas [24]. 6TiSCH minimal configuration is the standard strategy for the allocation of cells for the control frame transmission [21]. In cases where one shared cell is not enough especially for dense or large networks, extra cells can be allocated as proposed in [25]. Also, the frequency of the control messages can be optimized taking the number of neighbors into account [26], [27].

Orchestra [28] and ALICE [29] are autonomous scheduling mechanisms, which are not designed with a focus on the network formation. These mechanisms build a schedule consisting of a set of slotframes dedicated for MAC, routing and application layers separately. While Orchestra run on a single channel, ALICE uses three different channel offsets for three slotframes. These mechanisms are not designed from a frequency diversity perspective. However, these mechanisms can be utilised for allocating cells between individual pairs of nodes using nodes' identifiers as proposed in this paper.

\section{Steerable ANTENNA MOdel}

In this study, a new antenna model is created to emulate the use of steerable antennas in 6TiSCH networks. The radiation pattern of the steerable directional antenna is given in Fig. 1(a). The maximum gain of the antenna is assumed to be $5 d B i$ according to [6], [16], [30]. Gain of the receiver antenna is calculated according to (1).

$$
G(\theta)[d B]=10(1+\cos (\theta))-15 .
$$

where $\theta$ is the angular difference between the radiation direction of the transmitter and receiver antennas. 10 and 15 values are chosen considering radiation pattern which is given in Fig.1(a). Simulated antenna increases the transmission range around 30 meters at $0^{\circ}$ by providing $5 d B$ gain, and has around 40 meters shorter range at $180^{\circ}$ as shown in Fig.1(b). Transmission range is calculated according to (2) where antenna gain is added to the standard path loss formula.

$$
R S S=P_{T X}+P_{P L}-10 K \log _{10}\left(\frac{d_{12}}{d_{0}}\right)+G\left(\theta_{12}\right) .
$$

where $P_{T X}$ is the transmission power, $P_{P L}$ is the path loss at the reference distance $d_{0}, K$ is the path loss exponent, $d_{12}$ is the distance between nodes 1 and 2, $G\left(\theta_{12}\right)$ is the directional gain of the receiver antenna as given in (1). The receiver sensitivity level used in the evaluation of the proposed mechanism is chosen according to the TI exp5438 platform which comes with a CC2420 radio which has as sensitivity level of $-95 \mathrm{dBm}$

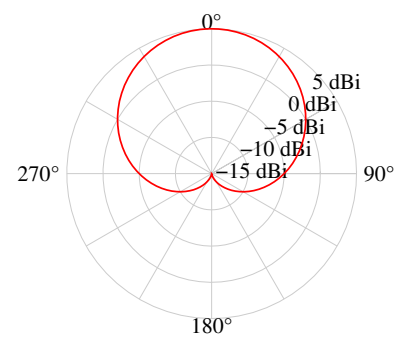

(a) Radiation pattern

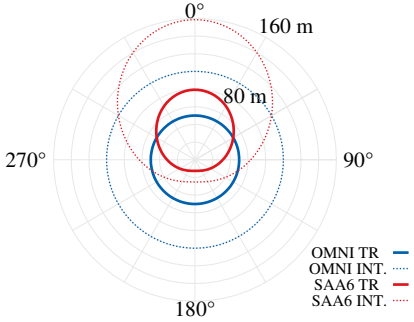

(b) Transmission/Interference range
Fig. 1. Simulated radiation pattern, and transmitting/interference range according to the active direction of the antenna.

2. Based on [31], the reference distance $\left(d_{0}\right)$ is set to 1 meter, the reference path loss is set to $-52 \mathrm{dBm}$, and the path loss exponent is set to 2.5 .

\section{IMPROVING NETWORK FORMATION}

In this section, methods for improving network formation are introduced.

\section{A. Neighbor Direction Estimation}

A node, which has a steerable directional antenna, must turn its antenna in the right direction prior to communication. Therefore, nodes should know in which direction they shall point their antennas to reach their neighbors. In this section, an RSSI based neighbor direction estimation mechanism is outlined. The best direction for each neighbor is kept in a list, and this list is updated considering the RSSI values retrieved from the physical layer for every successful frame exchange. The details of the algorithm are given in Algorithm 1 which is called only after receiving a packet.

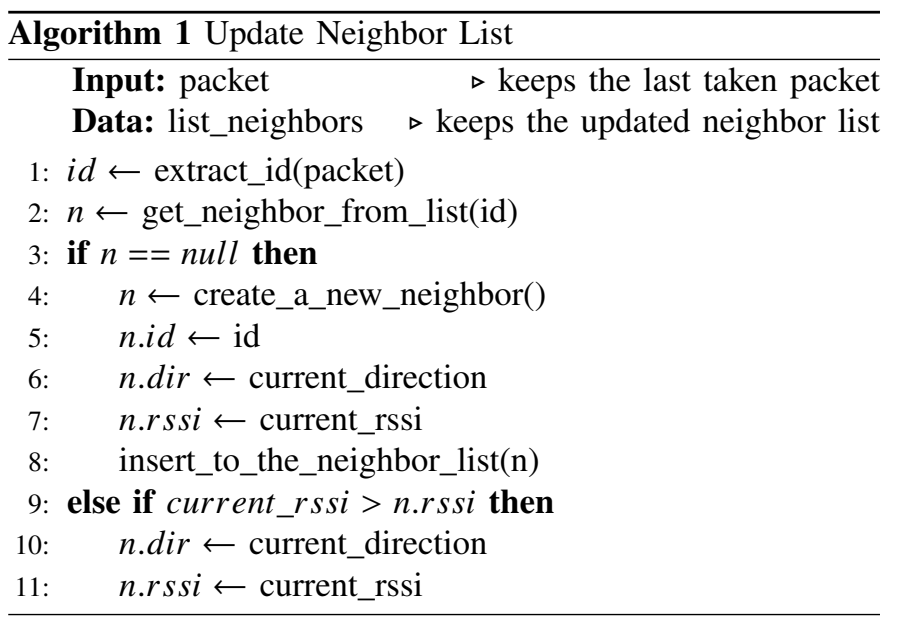

During the network discovery, nodes set their antennas toward a random direction at the start of each slotframe rather than following a steering pattern or configuration. Therefore, it is not possible to define an upper bound on how long the discovery stage will take. Furthermore, nodes may choose nonoptimal parents for a certain period of time until the network

${ }^{2}$ CC2420 radio [online]. Website http://www.ti.com/product/cc2420 [accessed May 2021] 
reaches a stable stage. After receiving a frame from a neighbor, the address of the neighbor, the current antenna direction, and the last measured RSSI values are stored in a table. The direction information is updated for every successful frame exchange between neighboring nodes. And the largest received RSSI value represents the best direction to communicate with a neighbor. When a new packet is received, the last RSSI value is compared with the recorded largest RSSI value for the neighbor and the direction information is updated.

During the synchronisation phase, the antenna direction is changed randomly for each slotframe. Due to the narrow antenna coverage area, neighbor discovery process takes longer as compared to the omnidirectional antenna solution. On the other hand, the synchronisation time can be shortened by allocating autonomous cells through which multicast messages are duplicated to provide a better opportunity for the near by nodes to get synchronised to the network.

The ability to dynamically steer the antenna and transmit in the desired direction can improve the communication performance of the node as long as the radiation direction is chosen properly. The selected antenna direction toward a particular neighbor may not be stable over time due to variations in the wireless communication channel. Therefore, adaptive protocols are required to dynamically change the best radiation direction according to the channel conditions. Algorithm 2 presents a mechanism that updates the best radiation direction dynamically for the destination node. To achieve this goal;

- Transmitter node transmits the frame by setting the antenna direction toward the best direction of the intended destination. Please note that the direction of the destination is kept in the neighbor table which is needed by the unicast traffic.

- The receiver node sets its antenna direction to the best direction but with an angular margin (i.e., from $-15^{\circ}$ to $+15^{\circ}$ )

- The receiver node updates its neighbor table, if the new direction provides a better RSSI value as compared to the current direction as given in Algorithm 1.

- Antenna direction is set to a random direction in shared cell since multicast frames are transmitted without the knowledge of the receiver.

Data packets generally consist of unicast messages transmitted from the child node towards its parent. However, multicast messages should be received by all of the neighbors of the transmitting node. Therefore, multicast messages provide an opportunity for the transmitting nodes to discover the direction of their neighbors via the allocated autonomous cells. As a result, this repetitive process enables nodes to adjust their radiation direction to increase the their radio link qualities.

The RSSI value changes with the relative directions of the transmitter and receiver antenna pairs. Fig. 3 shows the RSSI change over time for four pairs of nodes where the topology of the network for the selected pairs of nodes is given in Fig.2. The two reference lines in the figure present (a) if omni-directional antenna is used, (b) if directional antenna is used, but they point directly at each other (best case). Initial RSSI values for the communicating pairs of the nodes are greater than $-95 \mathrm{dBm}$, since the receiver sensitivity value of
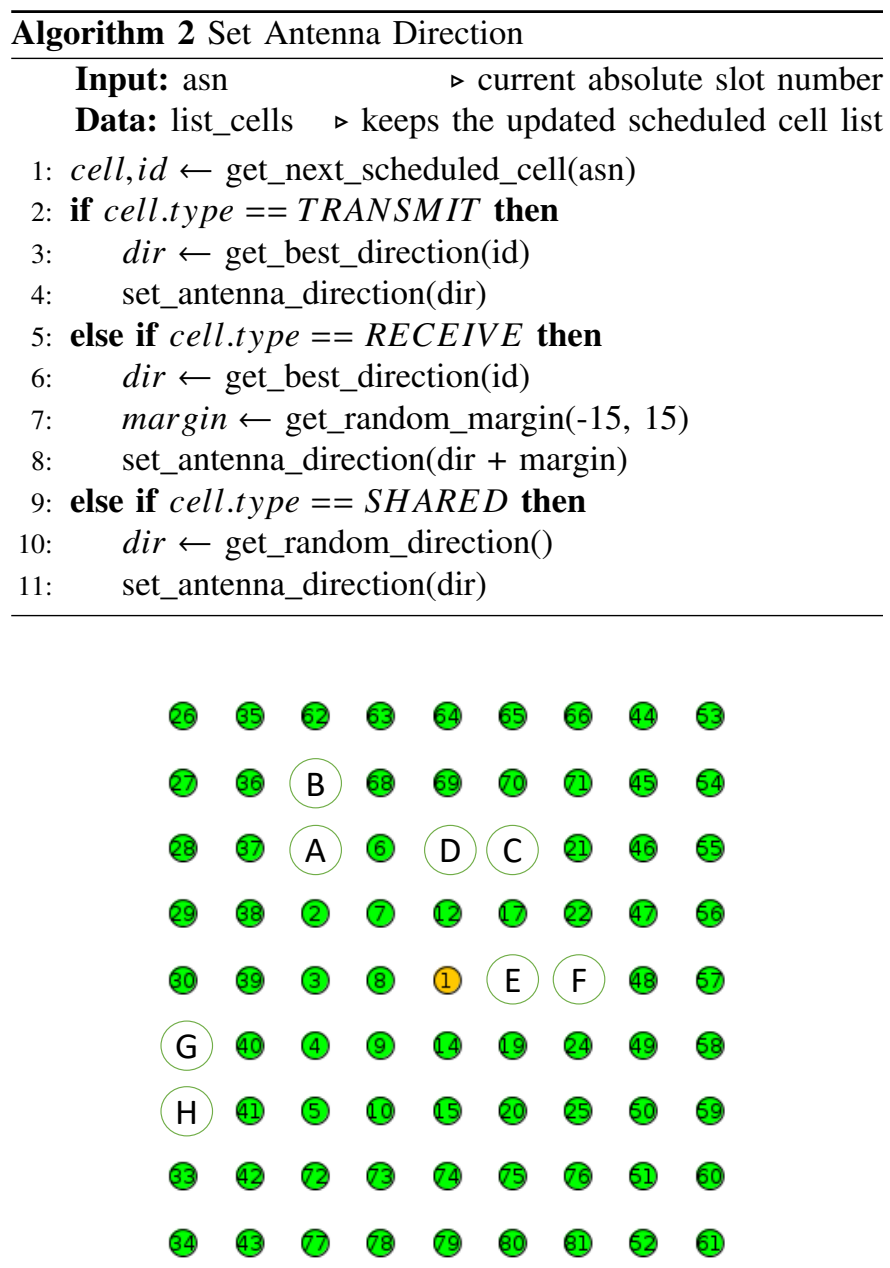

Fig. 2. Network topology used during the Cooja based performance evaluations.

the radio is set to this value. However, initial RSSI value differ for each pair due to both their relative positions in the network and their initial antenna orientations. The nodes in the simulated scenario are located at $40 \mathrm{~m}$ equal distance to each other in a grid formation. Therefore, RSSI values converges to $-82 \mathrm{dBm}$ according to RSS equation in (2) indicating that the optimal antenna orientations have been established after a certain discovery time period. As it can be seen from the Fig. 2, the node pairs improve their observed RSSI values over time by adjusting their antenna directions using the algorithm presented in (2).

\section{B. Utilizing Direction Information in Shared Cells}

Three conditions must be satisfied by the two nodes equipped with steerable directional antennas for a successful communication, where $\mathrm{X}$ shows the transmitter and $\mathrm{Y}$ shows the receiver node.

- Node X must transmit in the direction of Node Y,

- Node Y must listen in the direction of Node X,

- No other node must transmit in the direction of Node Y on the same frequency channel.

These conditions can be satisfied by building a proper schedule. In the case of omni-directional antenna, the receive 


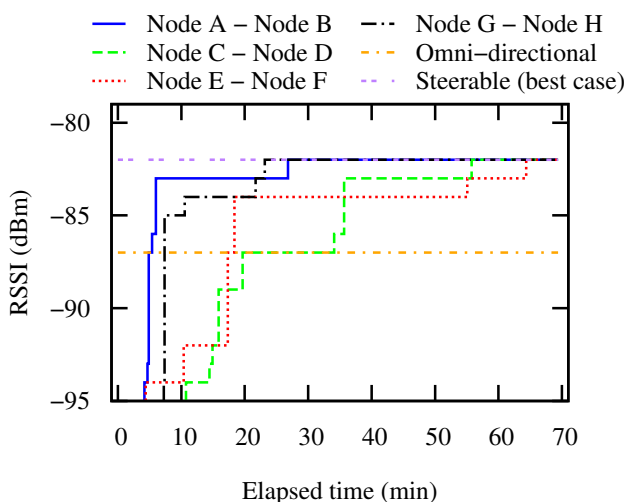

Fig. 3. RSSI v.s. Elapsed time. (According to topology given in Fig.2)

probability of a neighboring node within the range of the transmitting node can be assumed to be $100 \%(P(R)=1)$ provided that there is no interference in the communication medium. For the steerable antenna case, each node must cover full $360^{\circ}$ area to find a feasible neighbor to communicate with. However, the nodes must select random directions to broadcast beacons during the discovery phase. In this case, the EB reception probability of a node becomes $P(R)=\frac{\alpha}{2 \pi} \frac{\beta}{2 \pi}$, where $\alpha$ and $\beta$ are the beamwidths of the transmitter and receiver antennas, respectively.

Following the discovery phase, nodes at the edge of the network will have neighbors towards the direction of the network coordinator (i.e. center of the network). Such nodes are named as "leaf nodes" and they do not need to scan the whole $360^{\circ}$. In this case, the receive probability of an RPL parent node can be estimated as $P(R)=\frac{\alpha}{2 \pi} \frac{n}{n+1}$ for the leaf nodes, where $n$ is the number of neighbors. Of course, the leaf node may need to point its antenna toward a random direction occasionally to let new nodes to join the network. $(n+1)$ is used to factor this in to the analysis.

\section{Transmitting Broadcast Messages via Dedicated Cells}

Broadcast messages such as EB, DIO, and DIS are transmitted over the shared cells in 6TiSCH protocol. Furthermore, these cells are also used for the initial 6P negotiations [9]. Therefore, shared cells are indispensable resources for the network formation, responsible for the transmission of important control messages.

Integration of a steerable directional antenna to 6TiSCH protocol poses new challenges since a node can not reach all of its neighbors in the shared cell due to the directional coverage of the antenna as given in Fig.1(a). To overcome this challenge, one solution is to increase the number of shared cells by $\frac{2 \pi}{\alpha} \frac{2 \pi}{\beta}$. In this case, each neighbor is covered with a shared cell dedicated to the direction of the neighbor. Predictably, this solution makes inefficient use of the bandwidth and energy resources. Another solution to this challenge is to allocate additional dedicated cells between neighbors instead of shared cells. Dedicated cells can be negotiated between the nodes and they provide efficient use of resources since they are expected to be collision free. When a node transmits a broadcast frame over the dedicated cell, the broadcast message is kept in the MAC buffer until the node sends the broadcast frame towards all of its neighbors via the dedicated cells.

\section{Autonomous Scheduling of Broadcast Messages}

Two slotframes with different lengths which are mutually prime are created for the proposed solution. One of the slotframes is used for data traffic, and the other one is used for the control messages, which is shown in detail in Fig.4.

A simple topology consisting of 4 nodes is given in Fig.4(a). The slotframe created by the distributed scheduling algorithm for this topology is shown in Fig.4(b). Blue color indicates the cells scheduled for transmission, and green color indicates the cells scheduled for reception. Since these dedicated cells are scheduled for the pairs of nodes, they create a reliable and interference free communication link between these nodes. The red colored cells represent the shared cells, and provide contention based access to the medium. Since the directional antenna cannot transmit omnidirectionally, a second slotframe is needed to be able to transmit multicast messages to all neighboring nodes. The slotframe generated autonomously is given in Fig.4(c).

Autonomously scheduled slotframe is used for control messages and does not require negotiations with the neighboring nodes. Instead, it relies on (3) to select a timeslot and a channel offset to transmit towards a neighboring node. The slotframe used for data traffic is scheduled by the Scheduling Function Zero (SF0) as introduced in the IETF 6TiSCH standard.

$$
\begin{aligned}
& \operatorname{slot}_{T X}(n)=\bmod \left(\text { hash }\left(I D_{n}, I D_{o}\right), L_{\text {auto }}\right) \\
& \operatorname{slot} t_{R X}(n)=\bmod \left(\text { hash }\left(I D_{o}, I D_{n}\right), L_{\text {auto }}\right) \\
& \operatorname{channel}(n)=\bmod \left(\text { hash }\left(I D_{o}, I D_{n}\right), L_{\text {hop }}\right)
\end{aligned}
$$

where $I D_{n}$ is the neighbor's id, $I D_{o}$ is the own id, $L_{\text {auto }}$ is the autonomous slotframe length and $L_{h o p}$ is the length of the channel hopping sequence.

When a node is associated with the network, it allocates the necessary cells by looking at its neighbor table. Here, each node autonomously allocates one Tx and one Rx cell for each of its neighbors. This process is summarized in Algorithm 3. Moving the shared cell traffic to dedicated cells in this manner provides a new approach as compared to literature [9], [18], [21], [23].

\section{E. Resource Usage Analysis}

A section of the network topology used in the test scenario is given in Fig.5. As seen in the figure, the same network has fewer number of hops when nodes are equipped with steerable antennas. Establishing connectivity with the RPL Root node with fewer hops implies that fewer cells are needed for communication leading to a better utilisation of the bandwidth resources. Furthermore, directional antennas have better interference suppression properties as compared to omni-directional antennas meaning that fewer frequency channels can be used in the network.

In the evaluation scenario, each node produces data packets periodically and transmits them to the RPL Root. So, each 


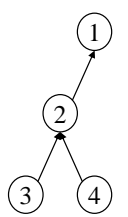

(a) Topology

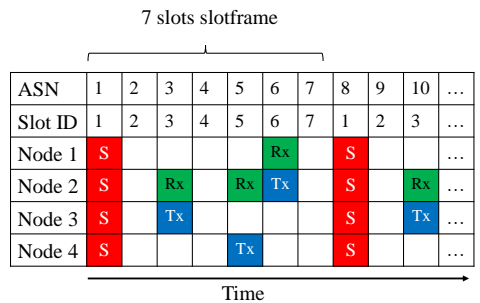

(b) Slotframe for data traffic (SF0)
19 slots slotframe

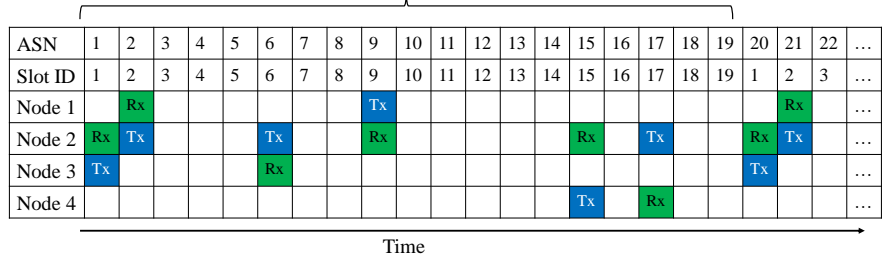

(c) Slotframe for control messages (Autonomous)

Fig. 4. Illustration of the slotframes generated by SF0 and autonomously, in a 4-node network topology.
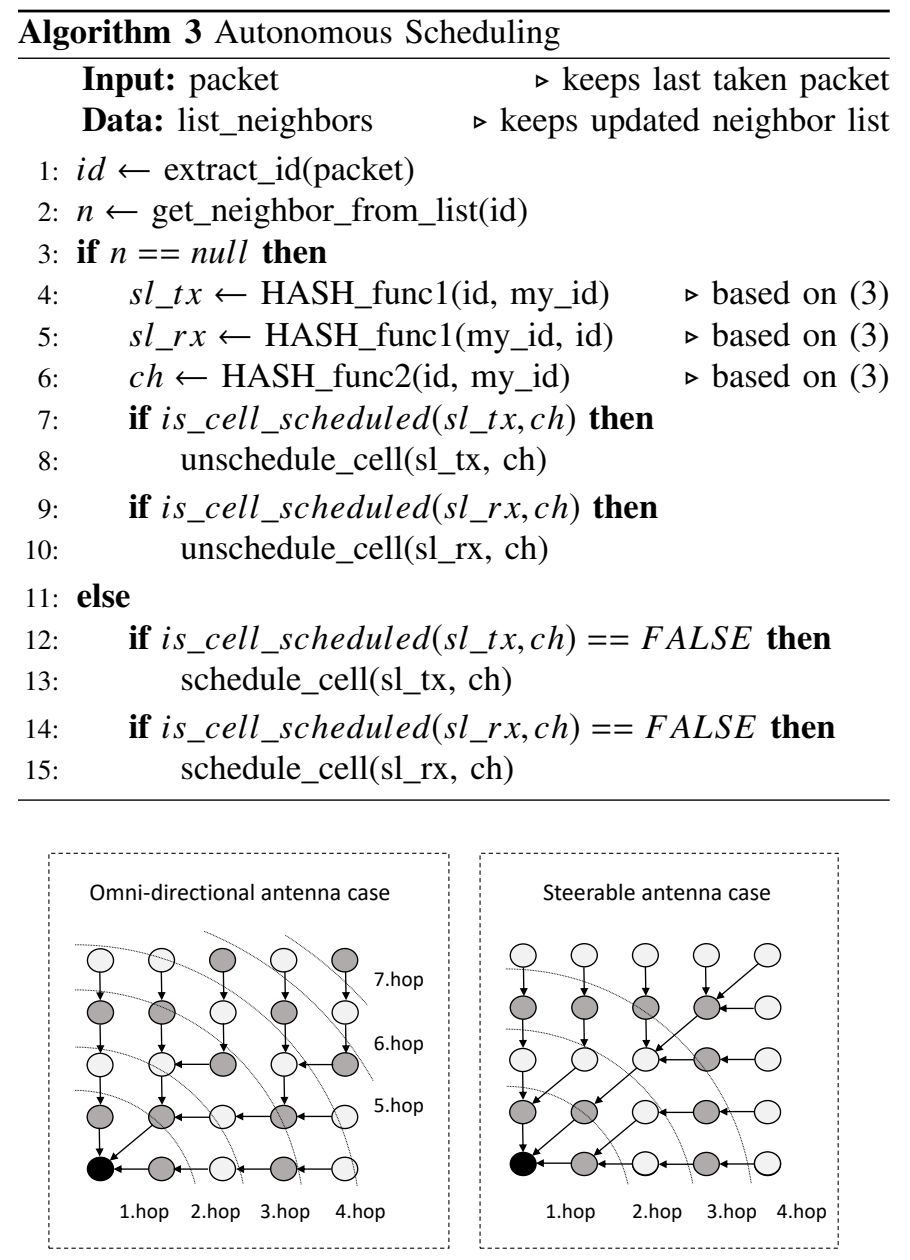

Fig. 5. A section of the 81 node network topology used in the performance evaluations of the proposed solution. The black node is the RPL Root located at the center of the network. White and grey colored nodes represent different hop levels.

node requires to allocate a number of Tx cells for these transmissions as given in (4).

$$
C=\left\lceil\left(D_{t} L_{s}\right) / I_{t}\right\rceil
$$

where $D_{t}$ is the time slot duration, $L_{s}$ is the data slotframe length (slotframe which is used for the transmission of data packets), $I_{t}$ is the transmission interval (indicates the data generation period), and $C$ is the cost which shows the number of required cells for each node to handle the generated data traffic. The total number of Tx cells required by all of the nodes at any hop level is given in (5).

$$
\begin{aligned}
& C_{h(x)}=N_{h(x)} C \\
& C_{h(x-1)}=C_{h(x)}+N_{h(x-1)} C
\end{aligned}
$$

where $C_{h(x)}$ is the total number of the required Tx cells for the nodes at hop level $x, N_{h(x)}$ is the number of nodes at hop level $x$. Therefore, the total number of Tx cells allocated for the whole network can be found as given in $(6)^{3}$.

$$
C_{s}=\sum_{i=1}^{x} C_{h(i)}
$$

The total number of Tx and Rx cells should be proportional since each node is required to act as a relay in the network. In addition to standard shared cells, SAA6 solution makes use of autonomously allocated cells for broadcast messages to speed up the synchronisation process. The number of extra cells allocated by autonomous scheduling function per node is given as (7),

$$
C_{a}=\frac{2 N_{n} L_{s}}{L_{a}}
$$

where $N_{n}$ is the number of 1-hop neighbors, $L_{s}$ is the slotframe length which is used for the data traffic, and $L_{a}$ is the slotframe length which is used by the autonomous scheduling function. For each neighbor, $1 \mathrm{TX}$ and $1 \mathrm{RX}$ cells are allocated to enable a faster network synchronisation by transmitting copies of the control frames via these extra cells. Since the autonomous slotframe length is larger than that of the data slotframe length, we divide $L_{s}$ by $L_{a}$ when calculating the cost of allocating these extra autonomous cells.

The cost introduced by the extra cells in relation to the cells allocated by the standard 6TiSCH scheduler can be calculated using (8). The number of cells required for the steerable antenna solution can be calculated as the sum of $C_{s}$ and $C_{a}$. However, omni-directional antenna solution does not require the $C_{a}$ cells.

$$
C_{t}=\left\{\begin{array}{lll}
C_{s} & \text { if } & \text { omni } \\
C_{s}+C_{a} & \text { if } & \text { steerable }
\end{array}\right.
$$

As shown in the example scenario presented in Fig.5, each node in the network except leaf nodes has a total number of 8

\footnotetext{
${ }^{3}$ Please note that the topology requires a converge-cast traffic pattern having a negative impact on the nodes closer to the RPL Root.
} 
TABLE I

COST ANALYSIS

\begin{tabular}{|l|c|c|c|}
\hline$I_{t}$ & $C_{t}^{\text {omni }}(\mathbf{8})$ & $C_{t}^{\text {steer }}(\mathbf{8})$ & $C_{t}^{\text {steer }} / C_{t}^{\text {omni }}$ \\
\hline 1 & 296 & $240+80=320$ & 1.08 \\
\hline 0.5 & 592 & $480+80=560$ & 0.94 \\
\hline
\end{tabular}

neighbors. For each neighbor, $1 \mathrm{Tx}$ and $1 \mathrm{Rx}$ slots are allocated in the autonomous slotframe. Therefore, each node needs additional 16 slots for SAA6 mechanism to make the discovery process quicker. Of course, the cost of introducing extra cells to the network to speed up the synchronisation process will vary depending on the topology of the network. The topology in this section is used as an performance indicator for the real life networks. In the evaluation tests, autonomous slotframe length is taken as 397 and data slotframe length is taken as $51^{4}$.

Since the steerable antenna can reach the root node with fewer number of hops due to higher antenna gains toward the receiving node, a system utilizing steerable antennas needs fewer cells compared to the omnidirectional antenna solution. In this case, the required number of cells for the network formations is reduced about $25 \%$ as indicated by (5) and (6). However, the steerable antenna solution presented here needs a fixed number of additional autonomous cells regardless of the transmission interval to speed up the synchronisation process as shown in (8).

When the transmission interval is greater than $750 \mathrm{~ms}$, each node must allocate minimum one cell due to the ceiling function in (4). As the transmission interval value gets smaller, the cost of autonomous cells decreases, since $C_{a}$ values is proportional to the network size only. When the transmission interval is chosen as 1 second, the required number of cells for the steerable antenna solution is about $8 \%$ more than that of the solution utilising omnidirectional antennas. But, for the presented topology, when the transmission interval is set to 0.5 second, the number of the cells required by the proposed solution is $6 \%$ less than that of omnidirectional solution due to the gains achieved by the reduced hop numbers in the network as presented in Table I. In summary, the proposed solution provides an efficient solution for networks with a heavy network traffic.

\section{Performance Evaluation}

Contiki $\mathrm{OS}^{5}$ is used to evaluate the performance of the SAA6 mechanism. Contiki OS supports IETF 6TiSCH protocol stack, and works integrated with a network simulator called COOJA which simulates the radio medium and allows the emulation of firmwares produced by several toolchains. COOJA simulator supports omni-directional antennas by default [31]. Therefore, the total received signal strength (RSS)

\footnotetext{
${ }^{4}$ There is not a standard value for the length of a slotframe. It is chosen according to network topology and traffic needs to provide minimum network latency and energy consumption. When multiple slotframes are used together, their lengths need to be prime among themselves so that they do not overlap often. In case they overlap, autonomous slots have higher priority in the proposed solution.

${ }^{5}$ Contiki: The Open Source OS for the Internet of Things [online]. Website http://www.contiki-os.org [accessed May 2021]
}

TABLE II

TEST PARAMETERS

\begin{tabular}{|l|l|}
\hline Parameter & Value \\
\hline Slotframe length & 51 \\
\hline Autonomous Slotframe length & 397 \\
\hline Timeslot duration & $15 \mathrm{~ms}$ \\
\hline Shared cell count (omni) & 3 \\
\hline Shared cell count (steerable) & 1 \\
\hline Transmit range (omni) & $50 \mathrm{~m}$ \\
\hline Interference range (omni) & $100 \mathrm{~m}$ \\
\hline Transmit range (steerable) & from 15 to $80 \mathrm{~m}$ \\
\hline Min EB period & $16 \mathrm{~s}$ \\
\hline Max EB period & $50 \mathrm{~s}$ \\
\hline Min DIO interval & $4.096 \mathrm{~s}$ \\
\hline Max DIO interval & $1048.576 \mathrm{~s}$ \\
\hline Inter-node distance & $40 \mathrm{~m}$ \\
\hline Network dimension & $320 \mathrm{~m} \mathrm{x} \mathrm{320} \mathrm{m}$ \\
\hline Test duration & 2 hours \\
\hline
\end{tabular}

value calculation in COOJA has been adapted using the path loss formula given in (2) to create a deterministic path loss model.

A modified COOJA simulator with steerable support is used for the performance evaluation of the proposed solution whose performance is analyzed for three distinct scenarios; (1) Nodes are equipped with only an omni-directional antenna (base scenario), (2) Nodes are equipped with a steerable directional antenna and transmit broadcast messages in a random direction, (3) Nodes are equipped with a steerable directional antenna and transmit broadcast messages over autonomously allocated cells as unicast by setting the antenna in the best direction (SAA6 mechanism). The performance results are presented in terms of synchronization latency, shared cell collision rate, packet delivery ratio, and energy consumption metrics.

\section{A. Network Topology and Assumptions}

In this section, the network topology and the assumptions used in the test scenarios are introduced.

- Each node is assumed to be static, and equipped with only one radio having a fixed transmit power $(0 \mathrm{dBm})$. At any time, a node can either be only in transmit or listen mode, but not both.

- Each node is equipped with a steerable directional antenna, that can scan $360^{\circ}$ by pointing the antenna at different directions.

- Root node is equipped with three switchable directional antennas [13], where each of them can scan $120^{\circ}$ area. All of the switchable antennas can be activated to emulate an omni-directional antenna behaviour.

- The parameters used in the tests are given in Table II.

\section{B. Synchronization Latency Tests}

Synchronization time is the elapsed time from the transmission of the first EB frame from the root node until the synchronization of the entire network. At this time, all nodes would have received at least one EB frame, and get synchronized to its time source neighbor by learning the minimal schedule. 


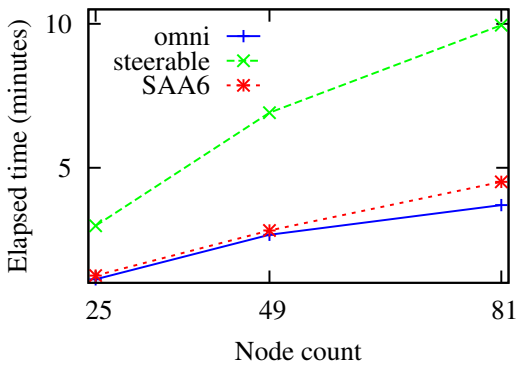

(a) Synchronization latency

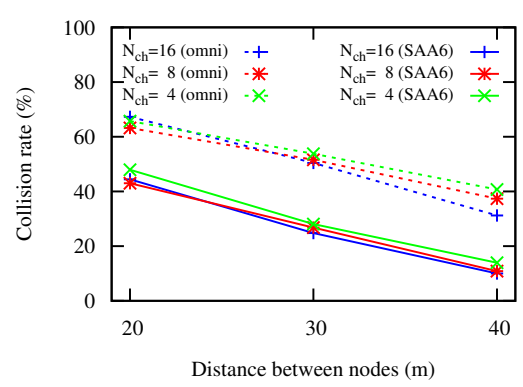

(b) Collision rate

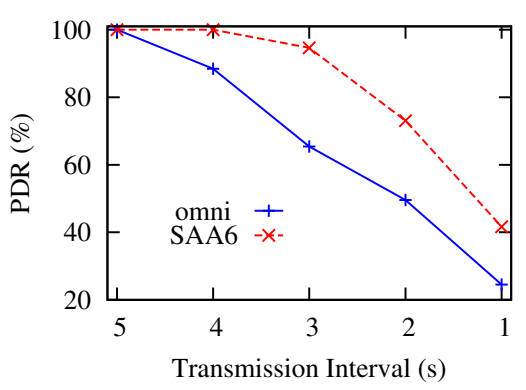

(c) Transmission Interval v.s. PDR

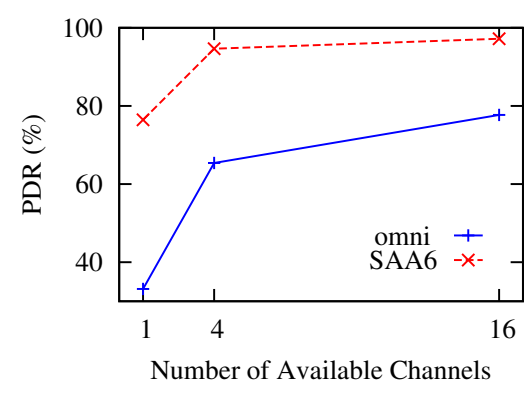

(d) Frequency Channels v.s. PDR

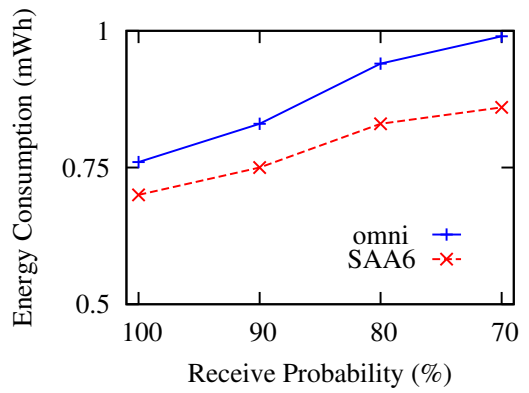

(e) Energy consumption

Fig. 6. Results for 81 Node Network

Fig.6(a) shows the variation of the synchronization time for different antenna configurations with growing network size when the number of available channels is set to 4 . As expected, the required time to achieve network synchronization increases with the network size, but at different rates for each configuration. Omni and SAA6 mechanisms present a lower synchronization time where SAA6 achieves a faster network synchronisation as compared to the pure steerable antenna solution at the expense of slightly increased energy consumption due to autonomous cells. The standard solution makes use of an omni-directional antennas. On the other hand, the steerable antenna solution can not achieve the same synchronization performance due to its narrow coverage area in the neighbor discovery phase. The proposed SAA6 solution achieves a better performance as compared to the standard steerable antenna solution since it transmits the control messages multiple times over the allocated autonomous cells.

In this case, SAA6 shows similar performance with the solution utilizing omni-directional antennas. The increasing number of neighbors creates an opportunity for the nodes equipped with omni-directional antennas to receive broadcast messages. Unfortunately, steerable antenna solutions do not scale as well as networks utilising omni-directional antennas due to scanning latency attached to directional antennas. The simulation results clearly indicate that the SAA6 scheme performs better than the pure steerable antenna solution which does not use Algorithm 1 and Algorithm 2, and improvement is more prominent for the larger networks.

\section{Collision Rate Tests}

As shown in the Fig.6(b), steerable antennas decrease the collision probability more than $20 \%$ in the shared cell without the need for an extra method such as adjusting EB interval dynamically. The collisions have a negative impact on the synchronization times. The results indicate that it is possible to significantly improve the shared cell success probability by employing directional antennas.

Since all synchronized nodes use the same frequency channel in the shared cell, the number of channels does not have a considerable impact on the collision rate. However, the contention in the shared cell increases in dense networks resulting in an increased collision rate. From the analytical and simulation results, it is observed that smart antenna solutions reduce the collisions in the shared cells of the 6TiSCH networks significantly especially in scenarios with high network density.

\section{Packet Delivery Ratio Tests}

Fig.6(c) shows the Packet Delivery Ratio (PDR) results for different transmission intervals when a total of 4 frequency channels are utilized. Lower PDR values are observed for shorter transmission intervals due to the lack of resources according to (4). SAA6 provides up to $30 \%$ higher PDR for the evaluated scenarios, since it utilizes spatial separation provided by steerable antennas. Fig.6(d) shows the PDR for different number of available frequency channels when a fixed transmission interval of 3 seconds is used. When the number of available frequency channels is limited, it is possible that the scheduler allocates cells with the same channel offsets. In this case, the simultaneous transmissions interfere each other more often. On the other hand, having a smart steerable antenna solution creates a third dimension that cell resources can be scheduled in allowing a higher throughput in the network. 


\section{E. Energy Consumption Tests}

Fig.6(e) presents the average energy consumed at each node. The receive probability in the figure presents the probability of a node receiving a frame destined to itself and this value changes according to the distance between the source and destination nodes. At the maximum distance, where the node is withing the communication range, the receive probability is at its minimum. On the other hand, if the source and destination nodes are next to each other, the receive probability becomes $100 \%$. SAA6 provides up to $20 \%$ energy saving due to increased communication range which improves the receive probability.

\section{CONClusion AND Future Work}

In this paper, adaptation of an electronically steerable antenna to $6 \mathrm{TiSCH}$ protocol is investigated by considering performance metrics such as synchronization time, PDR, and energy consumption. The $6 \mathrm{TiSCH}$ protocol is modified to accomodate the steerable antenna solution. The proposed Steerable Antenna Agnostic 6TiSCH Solution (SAA6) is extensively evaluated and the results verify that SAA6 outperforms omnidirectional antenna based solution in all aspects especially in terms of reliability, and network throughput.

It is proved that $6 \mathrm{TiSCH}$ protocol has the potential to support directional and smart antennas. This study provides useful insights for the future industrial IoT applications utilizing directional communications. As part of the future work, simulation results will be validated on real hardware under real-life conditions. Determining the best direction will be improved by utilising machine learning algorithms. Also, integration of multi-path communication with the steerable antenna solutions promises to be a way forward to improve network latency which will be investigated as a future research topic.

\section{ACKNOWLEDGMENT}

This research is supported by the Scientific and Technological Research Council of Turkey (TÜBITTAK) Research Fund under project number 118E289.

\section{REFERENCES}

[1] D. Minoli, K. Sohraby, and B. Occhiogrosso, "Iot considerations, requirements, and architectures for smart buildings-energy optimization and next-generation building management systems," IEEE Internet of Things Journal, vol. 4, no. 1, pp. 269-283, 2017.

[2] T. Okazawa, J. Ma, N. Komuro, Y. Choi, Z. Li, T. Pei, and H. Sekiya, "An opportunistic directional mac protocol with pulse/tone exchange in wireless ad-hoc networks," Wireless Personal Communications, vol. 111, no. 2, pp. 1187-1205, 2020.

[3] A. Varshney, T. Voigt, and L. Mottola, "Using directional transmissions and receptions to reduce contention in wireless sensor networks," in Real-World Wireless Sensor Networks, K. Langendoen, W. Hu, F. Ferrari, M. Zimmerling, and L. Mottola, Eds. Cham: Springer International Publishing, 2014, pp. 205-213.

[4] M. R. Palattella, N. Accettura, X. Vilajosana, T. Watteyne, L. A. Grieco, G. Boggia, and M. Dohler, "Standardized protocol stack for the internet of (important) things," IEEE Communications Surveys Tutorials, vol. 15, no. 3, pp. 1389-1406, Third 2013.

[5] R. Musaloiu-E. and A. Terzis, "Minimising the effect of wifi interference in 802.15.4 wireless sensor networks," International Journal of Sensor Networks, vol. 3, no. 1, pp. 43-54, Dec. 2008.
[6] M. Burtowy, M. Rzymowski, and L. Kulas, "Low-profile espar antenna for rss-based doa estimation in iot applications," IEEE Access, vol. 7, pp. 17403-17411, 2019.

[7] H. Liu, S. Gao, and T. Loh, "Electrically small and low cost smart antenna for wireless communication," IEEE Transactions on Antennas and Propagation, vol. 60, no. 3, pp. 1540-1549, March 2012.

[8] X. Liu, A. Sheth, M. Kaminsky, K. Papagiannaki, S. Seshan, and P. Steenkiste, "Dirc: Increasing indoor wireless capacity using directional antennas," in Proceedings of the ACM SIGCOMM 2009 Conference on Data Communication, ser. SIGCOMM '09. New York, NY, USA: Association for Computing Machinery, 2009, p. 171-182.

[9] X. Vilajosana, T. Watteyne, T. Chang, M. Vučinić, S. Duquennoy, and P. Thubert, "Ietf 6tisch: A tutorial," IEEE Communications Surveys Tutorials, vol. 22, no. 1, pp. 595-615, 2020.

[10] S. Duquennoy, A. Elsts, B. A. Nahas, and G. Oikonomo, "Tsch and 6tisch for contiki: Challenges, design and evaluation," in 2017 13th International Conference on Distributed Computing in Sensor Systems (DCOSS), June 2017, pp. 11-18.

[11] A. Aijaz, A. Stanoev, and U. Raza, "Gallop: Toward high-performance connectivity for closing control loops over multi-hop wireless networks," in Proceedings of the 27th International Conference on Real-Time Networks and Systems, ser. RTNS '19. New York, NY, USA: Association for Computing Machinery, 2019, p. 176-186. [Online]. Available: https://doi.org/10.1145/3356401.3356413

[12] T. Voigt, L. Mottola, and K. Hewage, "Understanding link dynamics in wireless sensor networks with dynamically steerable directional antennas," in Wireless Sensor Networks, P. Demeester, I. Moerman, and A. Terzis, Eds. Berlin, Heidelberg: Springer Berlin Heidelberg, 2013, pp. $115-130$.

[13] S. Gormus and S. Kulcu, "Enabling space time division multiple access in ietf 6tisch protocol," Turkish Journal of Electrical Engineering \& Computer Sciences, vol. 27, no. 6, pp. 2779-2791, 2019.

[14] A. Kausar, H. Mehrpouyan, M. Sellathurai, R. Qian, and S. Kausar, "Energy efficient switched parasitic array antenna for $5 \mathrm{~g}$ networks and iot," in 2016 Loughborough Antennas Propagation Conference (LAPC), Nov 2016, pp. 1-5.

[15] M. Rzymowski, P. Woznica, and L. Kulas, "Single-anchor indoor localization using espar antenna," IEEE Antennas and Wireless Propagation Letters, vol. 15, pp. 1183-1186, 2016.

[16] M. Rzymowski and L. Kulas, "Influence of espar antenna radiation patterns shape on ppcc-based doa estimation accuracy," in 2018 22nd International Microwave and Radar Conference (MIKON), May 2018, pp. 69-72.

[17] R. Alexander, A. Brandt, J. Vasseur, J. Hui, K. Pister, P. Thubert, P. Levis, R. Struik, R. Kelsey, and T. Winter, "RPL: IPv6 Routing Protocol for Low-Power and Lossy Networks," RFC 6550, Mar. 2012. [Online]. Available: https://rfc-editor.org/rfc/rfc6550.txt

[18] D. D. Guglielmo, S. Brienza, and G. Anastasi, "Ieee 802.15.4e: A survey," Computer Communications, vol. 88, pp. 1 - 24, 2016.

[19] L. Chen, Y. Li, and A. V. Vasilakos, "On oblivious neighbor discovery in distributed wireless networks with directional antennas: Theoretical foundation and algorithm design," IEEE/ACM Transactions on Networking, vol. 25, no. 4, pp. 1982-1993, 2017.

[20] B. El Khamlichi, D. H. N. Nguyen, J. El Abbadi, N. W. Rowe, and S. Kumar, "Collision-aware neighbor discovery with directional antennas," in 2018 International Conference on Computing, Networking and Communications (ICNC), March 2018, pp. 220-225.

[21] X. Vilajosana, K. Pister, and T. Watteyne, "Minimal IPv6 over the TSCH Mode of IEEE 802.15.4e (6TiSCH) Configuration," RFC 8180, May 2017. [Online]. Available: https://rfc-editor.org/rfc/rfc8180.txt

[22] Q. Wang, X. Vilajosana, and T. Watteyne, "6TiSCH Operation Sublayer (6top) Protocol (6P)," RFC 8480, Nov. 2018. [Online]. Available: https://rfc-editor.org/rfc/rfc8480.txt

[23] R. T. Hermeto, A. Gallais, and F. Theoleyre, "Scheduling for ieee802.15.4-tsch and slow channel hopping mac in low power industrial wireless networks: A survey," Computer Communications, vol. 114, pp. $84-105,2017$

[24] J. Vera-Pérez, D. Todolí-Ferrandis, S. Santonja-Climent, J. SilvestreBlanes, and V. Sempere-Payá, "A joining procedure and synchronization for tsch-rpl wireless sensor networks," Sensors, vol. 18, no. 10, 2018.

[25] C. Vallati, S. Brienza, G. Anastasi, and S. K. Das, "Improving network formation in 6tisch networks," IEEE Transactions on Mobile Computing, vol. 18, no. 1, pp. 98-110, Jan 2019.

[26] D. De Guglielmo, A. Seghetti, G. Anastasi, and M. Conti, "A performance analysis of the network formation process in ieee $802.15 .4 \mathrm{e}$ tsch wireless sensor/actuator networks," in 2014 IEEE Symposium on Computers and Communications (ISCC), June 2014, pp. 1-6. 
[27] M. Vučinić, T. Watteyne, and X. Vilajosana, "Broadcasting strategies in 6tisch networks," Internet Technology Letters, vol. 1, no. 1, p. e15, 2018.

[28] S. Duquennoy, B. Al Nahas, O. Landsiedel, and T. Watteyne, "Orchestra: Robust mesh networks through autonomously scheduled tsch," in Proceedings of the 13th ACM Conference on Embedded Networked Sensor Systems, ser. SenSys '15. New York, NY, USA: ACM, 2015, pp. 337350 .

[29] S. Kim, H. Kim, and C. Kim, "Alice: Autonomous link-based cell scheduling for tsch," in 2019 18th ACM/IEEE International Conference on Information Processing in Sensor Networks (IPSN), April 2019, pp. 121-132.

[30] H. Liu, S. Gao, and T. Loh, "Electrically small and low cost smart antenna for wireless communication," IEEE Transactions on Antennas and Propagation, vol. 60, no. 3, pp. 1540-1549, March 2012.

[31] J. Schandy, S. Olofsson, L. Steinfeld, and T. Voigt, "Improving sensor network convergecast performance with directional antennas." in EWSN, 2019, pp. 13-22. 